\title{
ILCEA
}

Revue de l'Institut des langues et cultures

d'Europe, Amérique, Afrique, Asie et Australie

$28 \mid 2017$

Passages, ancrage dans la littérature de voyage

\section{Des fleuves et des nations dans Seven Rivers of Canada (1963)}

of Rivers and Nations in Seven Rivers of Canada (1963)

\section{André Dodeman}

\section{OpenEdition}

\section{Journals}

\section{Édition électronique}

URL : http://journals.openedition.org/ilcea/4124

DOI : 10.4000/ilcea.4124

ISSN : 2101-0609

\section{Éditeur}

UGA Éditions/Université Grenoble Alpes

\section{Édition imprimée}

ISBN : 978-2-84310-374-2

ISSN : $1639-6073$

Référence électronique

André Dodeman, « Des fleuves et des nations dans Seven Rivers of Canada (1963) », ILCEA [En ligne] 28 | 2017, mis en ligne le 06 mars 2017, consulté le 30 avril 2019. URL : http://

journals.openedition.org/ilcea/4124; DOI : 10.4000/ilcea.4124

Ce document a été généré automatiquement le 30 avril 2019

(C) ILCEA 


\title{
Des fleuves et des nations dans Seven Rivers of Canada (1963)
}

of Rivers and Nations in Seven Rivers of Canada (1963)

\author{
André Dodeman
}

Bien que Hugh MacLennan soit plus connu aujourd'hui pour les sept romans qu'il publie entre 1941 et 1980, les nombreux essais et récits de voyage qu'il écrit restent une partie non négligeable de sa carrière d'écrivain. Au début des années 1960, MacLennan publie sept textes dans le magazine MacLean's qui seront ensuite réunis dans un ouvrage portant le titre de Seven Rivers of Canada. Les sept récits décrivent ses voyages sur les plus grands affluents et fleuves ${ }^{1}$ canadiens que sont le MacKenzie, le Saint-Laurent, la rivière des Outaouais, la rivière Rouge, la rivière Saskatchewan, le fleuve Fraser et le fleuve SaintJean. Ces textes sont en réalité le fruit d'une commande qu'il n'oublie pas d'évoquer dès l'introduction dans laquelle il parlera de son périple comme d'une véritable aventure personnelle dans l'espace et le temps. Ses voyages durent environ deux ans et demi, et les moyens de transport évoqués sont variés: il voyage à bord de plusieurs remorqueurs pour suivre le fleuve MacKenzie et il prend un avion pour découvrir la rivière Saskatchewan. MacLennan, qui a déjà une expérience dans l'enseignement de l'histoire de l'Antiquité grecque et romaine, se concentre sur les fleuves pour rappeler le rôle central qu'ils jouent dans la naissance, l'essor et le déclin des civilisations. Pour lui, l'essor des civilisations mésopotamienne et égyptienne ${ }^{2}$ aurait été impossible sans le Tigre, l'Euphrate et le Nil. À l'instar de ces civilisations du passé, l'histoire du Canada ne saurait faire abstraction de ces fleuves qui ont façonné le pays et facilité son peuplement. Le contexte des années 1960 joue un rôle central dans la lecture de ces récits, car l'auteur réagit fermement aux problématiques de cette période et aux défis futurs à relever. L'auteur déplore en effet la montée de mouvements nationalistes à l'aube du centenaire qui sera célébré en 1967 à Montréal, ville choisie pour organiser l'exposition universelle. Ce travail propose d'analyser les raisons pour lesquelles MacLennan choisit le récit de voyage, plutôt que le roman ou l'essai, pour défendre l'unité du pays face aux idées séparatistes. Dans un premier temps, ce travail traitera de la manière dont MacLennan construit sa propre figure de voyageur et de la place qu'il occupe dans une tradition 
canadienne du récit de voyage qui compte des auteurs de renom comme Susanna Moodie et sa sœur Catherine Parr Traill. Compte tenu de sa longueur, une attention particulière sera portée au premier récit intitulé «The MacKenzie ». Dans un deuxième temps, il sera question de la transformation du paysage dont l'esthétisation sert avant tout à éveiller un sentiment d'appartenance chez le lecteur en un territoire que l'auteur ordonne, quadrille et singularise en vue de construire une identité nordique. En dernier lieu, il s'agira de voir comment sa pratique de l'essai impacte ses récits de voyage. Le mythe national que MacLennan propose à ses lecteurs pour préserver l'unité du pays montre en effet à quel point toute tentative de définition de la nation reste sujette à controverse.

\section{L'écrivain voyageur}

2 Avant de publier Seven Rivers of Canada en 1963, MacLennan était déjà connu pour ses cinq romans qui visaient à décrire l'émergence d'une identité proprement canadienne. Pour lui, cette identité se construit principalement en se distinguant de l'empire britannique autrefois associé à la mère patrie et de son voisin américain devenu très puissant sur la scène internationale depuis la fin de la seconde guerre mondiale. Ces deux centres de gravité culturels et linguistiques n'ont pas facilité la tâche des écrivains canadiens anglophones qui voulaient marquer leur différence et ambitionnaient d'être lus dans le monde entier. Dans les nombreux essais qu'il publie, MacLennan se désigne volontiers comme un intellectuel et un écrivain engagé dont la fonction sociale consiste à s'impliquer dans les problématiques de son époque et à interpréter le monde pour mieux éclairer ses concitoyens. C'est dans l'optique d'éclairer les lecteurs sur la géographie du Canada que MacLennan décide de s'inscrire dans la tradition des récits d'exploration et de voyage qui ont marqué les débuts de l'historiographie canadienne. Cette historiographie comprend, entre autres, les récits d'exploration de Jacques Cartier qui entreprit plusieurs voyages au XVI ${ }^{\mathrm{e}}$ siècle, de Samuel de Champlain qui fonda la colonie du Québec en 1608, de Samuel Hearne qui remonta la rivière Coppermine pour atteindre l'Arctique en 1771 et d'Alexander MacKenzie qui atteignit l'océan Pacifique en 1793. Ces références, très présentes dans le texte, illustrent la dimension historique et encyclopédique du récit de voyage, et MacLennan use de ses connaissances et de son métier d'historien pour dresser un portrait épique des premiers explorateurs et emporter l'adhésion du lecteur au discours prônant l'unité politique du Canada.

3 Les sept récits de Seven Rivers of Canada sont précédés d'un essai paru en 1961 intitulé « The Rivers that Made a Nation ", dans lequel MacLennan écrit que l'écrivain est le mieux à même de lutter contre les idées reçues et de redorer l'image du pays :

If any modern Canadian is curious to know how his country was valued two centuries ago, all he need do is recall some of the sentiments it inspired among famous men at that time. Voltaire's dismissal of the Saint Lawrence Valley as "a few acres of snow" is almost too well known to repeat; it is less well known that Montcalm, who now is a Canadian hero, loathed the country he fought to defend. The British never valued Canada for herself. (1963: 9)

Ce passage fait état d'un passé marqué par un dénigrement du pays qui semble faire obstacle à tout enracinement possible dans le territoire et encourager les stéréotypes. Même le général Montcalm, le héros de la bataille des plaines d'Abraham qui a marqué la chute de l'autorité française en 1759 , ne fait pas exception à cette règle. En réponse à ce passé de clichés et pour contribuer à un sentiment national de fierté, MacLennan entreprend un voyage qui le conduira à sillonner le pays d'est en ouest en passant par ses 
grands fleuves. Comme MacLennan est connu jusque-là pour ses romans et ses essais, il doit d'abord se construire comme voyageur. Dans sa présentation de l'ouvrage collectif Écrire le voyage, György Tverdota rappelle que les récits de voyage « relatent les péripéties d'un déplacement, entrecoupées d'impressions et de réflexions» (Tverdota, 1994: vI). Bien que les sept récits de MacLennan soient bien le fruit de voyages, les péripéties de ces derniers ne sont véritablement décrites que dans le premier texte intitulé "The MacKenzie ».

5 Chacun des sept textes de MacLennan porte le nom du fleuve ou de la rivière qu'il est censé décrire, mais c'est dans le texte portant sur le fleuve MacKenzie que le voyage joue un rôle aussi important que celui des paysages. Pour l'auteur, la civilisation est née au bord des fleuves, si bien qu'ils finissent par devenir des symboles de l'origine et du destin d'un pays. William H. New, dans son article portant sur les œuvres de Hugh MacLennan et de John Mulgan, note l'importance que chaque auteur donne à la symbolique du fleuve : « In historical perspective, great rivers (e.g., the Tigris-Euphrates system, the Volga, the Ganges, the Seine) were associated with fertile valleys, settlements, and consequently, pockets of what has subsequently been referred to as 'civilization'” (New, 1995: 163) ${ }^{3}$. Suivre le cours du MacKenzie permet ainsi à l'auteur de voir où en est son propre pays dans l'histoire des civilisations.

6 «The MacKenzie » est un récit à la première personne et au passé qui relate son départ de l'est du Canada et son arrivée à Edmonton en avion pour entamer son voyage sur le fleuve. Les nombreuses escales qui ponctuent sa descente du fleuve sont autant d'occasions pour l'auteur de partager son expérience, ses impressions, les anecdotes et les témoignages glanés au fil des rencontres, ainsi que les explications historiques et didactiques censées permettre aux lecteurs de mieux saisir l'immensité géographique et symbolique de cet espace. À partir d'Edmonton, il lui faut prendre un avion et trois remorqueurs avant d'atteindre la petite ville d'Aklavik, un hameau qui se trouve aujourd'hui dans la région d'Inuvik en aval du fleuve. Entre chaque embarquement, les attentes sont longues (une semaine à Edmonton et une dizaine de jours à Fort Smith) et donnent lieu à des récits à caractère encyclopédique ayant trait à la profondeur et au débit du fleuve, à son histoire, ainsi qu'au portrait épique de l'explorateur qui lui a donné son nom. À Fort Smith, il se rend dans le camp de Bell Rock où il rencontre des travailleurs venus du monde entier. Il décrit leurs conditions de vie et va jusqu'à commenter leur régime alimentaire pour décrire l'ennui que peuvent causer les temps d'attente. À mesure qu'approche le départ tant attendu à bord du remorqueur Radium King, MacLennan adopte un ton de plus en plus didactique: il relate l'histoire de la compagnie de la Baie d'Hudson, explique le fonctionnement des remorqueurs et rapporte le discours des hommes rencontrés afin de souligner l'authenticité de son expérience. Le camp, comme l'auberge dont parle Jean Viviès (1999) dans Le récit de voyage en Angleterre au dix-huitième siècle, permet d'insérer le discours rapporté de l'autre et de dévoiler un espace intermédiaire, un espace d'attente censé préparer la prochaine étape du voyage.

7 L'étirement du temps de l'attente est propice à la réflexion et donc au récit. Robert Kroetsch, écrivain canadien connu pour son expérience du nord, écrit que le temps suspendu du nord canadien permet à l'écrivain de renouer avec une forme d'inaction qui encourage la réflexion et motive le récit :

In the North, people wait through whole seasons for freight to arrive; they wait years for governments to make decisions; they wait decades, or possibly centuries, for treaties to be signed. 
To wait is to alter violently the momentum and the purpose of Western culture. A condition generates its own system, its own values, and waiting becomes an alternate culture that is rich in reflection and meditation, those forms of inaction that become versions of action; waiting is a recovery of the eternal into our obsession with linear time. (Kroetsch, 1995 :

19)

MacLennan fait l'expérience de cette même atemporalité du nord canadien, une atemporalité qui devient la condition même du récit. Lorsqu'il est contraint d'attendre à Edmonton avant de s'envoler vers Fort Smith, il écrit que le nord et le sud se distinguent principalement par leurs temporalités: "In Edmonton, I soon made the discovery everyone makes the moment he has anything to do with the Canadian north. The time-sense I had grown up with ceased to mean anything in a country which, by southern standards, is essentially timeless " (1963: 36). C'est donc avec un regard de Canadien du sud, éduqué et cultivé, que MacLennan va construire sa vision du nord et la raconter. Pour ce faire, MacLennan recourt à son capital culturel pour traduire ses impressions lors d'un lever de soleil sur le Lac des Esclaves :

I woke at dawn wondering where I was, for a bright light was in my eyes. Never before or since have I seen such a sunrise. The sky over the river and land was a flat roof, livid and sinister, and it lay oppressively. In the east a blaze of orange had torn a jagged rent in the sky and the sunrise poured through between sky and water like a searchlight gone mad. It tore another rent in the west and travelled on into a sea of golden glory and the whole sky took fire all at once. A minute before it had been like the sky painted by El Greco over Toledo; now it was Turner's sky over the Thames estuary-but bigger, lonelier, more awe-inspiring. (1963: 53)

9 Ce passage à la première personne rappelle de nombreux textes romantiques dans lesquels la subjectivité de l'observateur occupe le premier plan. L'écrivain voyageur est ici submergé par l'expérience du sublime que suscitent les couleurs du ciel, ses jeux de lumière et la violence pénétrante du lever de soleil, sans parler de cet espace surdimensionné éclairé d'est en ouest par le soleil et dont Burke dit qu'il figure parmi les causes les plus puissantes du sublime (Burke, 1998 : 66). MacLennan n'a en effet d'autre choix que de recourir à des conventions littéraires européennes pour palier l'irréductibilité du nord au langage. Dans son ouvrage Analogie et récit de voyage, Alain Guyot rappelle que le relateur, ou le narrateur du voyage, « constate vite que ce qu'il a vu est irréductible au langage, ce qui rend au premier abord impossible toute description, toute tentative de faire voir " (2012: 29). La description qui est donc faite du nord canadien est eurocentrique, tout comme l'ont été les récits de Susanna Moodie publiés un siècle plus tôt. C'est en 1852 que Moodie publie les récits de son départ de l'Angleterre et de son arrivée au Canada sous le titre de Roughing It in the Bush. L'ouvrage s'adresse à un public anglais du XIx ${ }^{e}$ siècle et regorge de passages visant à esthétiser une nature définie en termes de grandeur et de puissance. Dès le second chapitre qui relate son trajet de Grosse Isle à Québec, Moodie ne cache pas son émerveillement à la vue des paysages et du fleuve Saint-Laurent :

Never shall I forget that short voyage from Grosse Isle to Quebec. I love to recall, after the lapse of so many years, every object that awoke in my breast emotions of astonishment and delight. What wonderful combinations of beauty, and grandeur, and power, at every winding of that noble river! How the mind expands with the sublimity of the spectacle, and soars upward in gratitude and adoration to the Author of all being, to thank Him for having made this lower world so wondrously fair-a living temple, heaven-arched, and capable of receiving the homage of all worshippers. (Moodie, 1852:28)

Comme le texte de MacLennan qui multiplie les références au sublime pour décrire un paysage dont la beauté transcende les mots, celui de Moodie rappelle le style britannique 
des $\mathrm{XVIII}^{\mathrm{e}}$ et $\mathrm{XIX}^{\mathrm{e}}$ siècles qui ont été marqués, entre autres, par le sentimentalisme et le romantisme. Son style traduit également son attachement à l'empire britannique et trahit son adhésion à une idéologie impériale qui conditionne les descriptions qu'elle fait du Canada et de sa population. Mais alors qu'elle décrit le paysage pour un lectorat anglais intéressé par l'expérience de l'émigration, MacLennan s'adresse à des lecteurs canadiens du sud du pays afin de les encourager à s'approprier un espace grandiose et à adhérer à sa vision de la nation. Cet espace nordique, constitutif de l'identité canadienne, devient celui de la nation tout entière. En le mesurant et en le quadrillant, l'espace devient territoire.

\section{De paysage en territoire}

11 L'une des techniques les plus utilisées par MacLennan pour apprivoiser ces espaces du nord est l'insertion de récits historiques qui les inscrivent et les soumettent à une temporalité humaine. Seven Rivers of Canada contient des récits de la colonisation du Canada : MacLennan y évoque les premiers explorateurs et les pères fondateurs tels que Jacques Cartier et Samuel de Champlain, ainsi que les aventuriers plus connus sous le nom de "coureurs de bois », ces commerçants de fourrure solitaires, surhumains et souvent francophones qui ont su s'adapter au climat, à la géographie et aux populations autochtones. Dans son article sur les voyages de MacKenzie, Robert Sayre parle de « récit synchronique " pour désigner ce type de récit enchâssé qui vise à synthétiser et à généraliser (2015: 47). Mais chez MacLennan, les récits et les événements qu'il décrit doivent être à la hauteur des espaces qu'il observe. Ils sont en effet accompagnés de contes populaires, plus connus sous le nom de tall tales en anglais, car l'auteur ne peut faire l'économie de l'exagération quand il s'agit de décrire une telle immensité géographique. Dans "The Rivers that Made a Nation», l'essai qui sert d'introduction à l'ouvrage, il écrit ceci :

Superlatives have bothered me all through the writing of this book, but I cannot avoid them without diminishing what seems to me the truth. Every new thing I have learned about the Canadian voyageur seems to me more incredible than the last. His deeds originated the Paul Bunyan myths of the American north-west, and Paul Bunyan was an inheritor of Hercules and Mercury in folklore. But the true and proved facts concerning the life of the voyageur are such that I can only say that if I, physically, am a man, he, physically, was a superman.

(1963: 21)

12 Les superlatifs et les contes - celui de Paul Bunyan est particulièrement révélateur ici sont précisément ce qu'il utilise pour exprimer l'immensité du Canada. S'ajoutent au mythe du voyageur les mythes plus classiques de l'Antiquité qui se déroulent dans ce «temps fabuleux des commencements » dont parle Mircéa Eliade dans Aspects du mythe (1963 :16). Cet essai annonce en effet que le «I» sera celui du conteur homérique qui relate les événements et les exploits des grands hommes du passé. Dans « The MacKenzie », suivre le cours du fleuve revient à revisiter et à relire, pour ne pas dire sacraliser, les récits de l'explorateur qui a appris à dominer le fleuve et à faciliter son peuplement. À l'instar des personnages légendaires qui peuplent les contes, seul un géant comme MacKenzie pouvait relever le défi du voyage sur le fleuve à la fin du XvIII ${ }^{\mathrm{e}}$ siècle : "The man [Alexander MacKenzie] who led this famous voyage of discovery has always seemed to me one of the most interesting and attractive personalities in Canadian history; in my opinion, this man is a giant among our people » (1963:57-58). Ces récits synchroniques ponctuent le voyage afin de doter le Canada d'une origine historique et de soumettre le fleuve à une chronologie humaine. 
Comme le fleuve MacKenzie, la nature que décrit l'auteur est ordonnée, jalonnée par le texte, et le récit synchronique qui relate le voyage de MacKenzie sert à capturer le cours du fleuve et à strier l'espace qui l'entoure. Comme le rappellent Deleuze et Guattari, l'espace strié, par opposition à l'espace lisse, est un espace que l'on mesure, un espace qu'on « compte pour l'occuper» (1980 : 447). Par conséquent, il serait plus juste de dire que MacLennan fait bien plus que visiter un espace, il l'occupe et l'ordonne en l'arpentant. La composante encyclopédique du récit de voyage se retrouve précisément sous la forme de mesures de volume, de profondeur, de surface et de débit. Afin de rendre justice au fleuve Saint-Laurent qui fait l'objet du second récit, MacLennan rappelle son lien avec les Grands Lacs dont il est l'émissaire : «For the Great Lakes contain an immense body of water. The maximum depth of Superior is 1,302 feet; of Huron 750; of Michigan 923; of Erie 210; of Ontario $774 »$ (1963: 68). Ces mesures de quantité ne visent pas uniquement à confirmer le regard du voyageur expert, puisque la connaissance de ces mesures lui permet également de s'approprier ces grands espaces et d'ordonner le chaos de la réalité. Ce quadrillage, comme l'explique Alain Guyot dans Analogie et récit de voyage, commence au XVIII ${ }^{\mathrm{e}}$ siècle à l'âge des grandes expéditions comme celles de James Cook. Il écrit qu' ' une grande partie des voyages à l'âge classique vont peu à peu devenir des instruments destinés à collecter et à mettre en ordre les données éparpillées sur la surface du globe, dans l'objectif de reconstruire l'unité de celui-ci sous la forme d'un véritable 'système de la nature'» (2012: 71). Quantifier l'espace revient donc à rationaliser la nature, à la ramener à des proportions humaines et à mieux la maîtriser. Cette mise en ordre chronologique et topographique permet à MacLennan de réaffirmer l'appartenance de ces espaces à un territoire national qui, comme le rappelle Edward W. Soja, se définit en termes de souveraineté, de propriété, de discipline, de surveillance et de juridiction ${ }^{4}(1989: 150)$.

14 À cela s'ajoute le temps géologique. Le texte de MacLennan regorge de données chiffrées qui renvoient à la fin de la dernière période glaciaire il y a plus de dix mille ans et à la naissance des fleuves et des Grands Lacs qui en a résulté. Tout comme Charles Lyell qui publie ses Principes de géologie dans les années 1830, MacLennan associe histoire et géologie par le biais de la centralité du regard de l'observateur éclairé. Ses observations permettent de remonter le temps et d'imbriquer l'histoire humaine dans une longue durée qui finit par justifier son existence. Les références à la géologie visent en quelque sorte à renouer le lecteur avec une certaine idée d'ancienneté de la culture canadienne qui pourrait rivaliser avec des histoires européennes plus longues et ainsi réorienter le regard du lecteur vers son propre territoire au lieu de chercher des origines ailleurs. Pour ce faire, il recourt à la sédimentation comme métaphore de la superposition, non de couches de sédiments, mais de récits et de peuples, et il écrit que les populations actuelles du Canada sont en réalité le résultat «des sédiments de l'histoire» (1963: 75). Cette vision découle aussi de ses lectures et de sa formation d'historien qui le conduiront à adhérer à la thèse selon laquelle l'histoire est constituée d'événements liés entre eux par des relations causales ${ }^{5}$. Ainsi les colons venus d'Europe s'insèrent dans une longue chaîne causale et trouvent tout naturellement leur place historique dans un temps géologique, l'intention étant bien entendu de légitimer leur présence en Amérique du Nord. Toutes ces données mettent l'accent sur la nordicité du pays et permettent d'inscrire le territoire dans une longue durée. Sherrill E. Grace, qui a écrit longuement sur la nordicité et le rôle qu'elle joue dans l'identité collective, affirme que c'est elle qui unit le pays et permet de dépasser les clivages linguistiques et culturels : 
Nordicity, after all, is one of the few things most Canadians (including today's Québécois) have in common. To celebrate the North as a symbol of national unity and Canadian identity is to make a virtue of geographical reality and socio-economic necessity, to differentiate us from the United States (becoming thereby a key component in an anti-continentalist rhetoric and political agenda), and to focus attention on larger, greater, more important and more abstract things than divisive regional concerns. (Grace, $2001: 67$ )

15 À l'heure où la province du Québec subit un changement profond de modèle économique et social qui attise les ardeurs nationalistes, MacLennan utilise le récit de voyage pour appeler à une unité nationale susceptible de transcender les divisions régionales $d u$ Canada. C'est pour cette raison que le voyage devient moins central au fil du texte et que le récit s'oriente progressivement vers l'essai.

\section{Une politique du voyage?}

16 Concernant la forme du récit de voyage, Jean Viviès rappelle deux différences majeures entre le récit de voyage et le roman, à savoir les rôles que jouent la causalité et le dénouement. À la différence du récit de voyage, la structure du roman est davantage "fondée sur un système de liens de causalité » (1999: 152) et s'achève avec un dénouement, là où le récit de voyage se clôt sur une terminaison (1999: 153). Ceci n'empêche en rien le voyageur de tirer ses propres conclusions et de décrire comment le périple l'a transformé. Alors que le récit du voyage sur le MacKenzie se termine sur un ton prophétique qui annonce le développement de la région au $\mathrm{xxI}^{\mathrm{e}}$ siècle, le second récit de voyage intitulé " The St. Lawrence » s'achève à Londres où l'auteur, au contact de cette ville qui reste pour lui l'une des plus importantes du monde, réalise l'importance qu'a jouée le fleuve dans l'histoire de son propre pays. Le Saint-Laurent est pour lui central, au point qu'il cite son propre roman Two Solitudes (publié en 1945) où le fleuve est désigné comme le carrefour historique du pays :

But down in the angle at Montreal, on the island about which the two rivers join, there is little of this sense of new and endless space. Two old races and religions meet here and live their separate legends, side by side. If this sprawling half-continent has a heart, here it is. Its pulse throbs out along the rivers and railroads; slow, reluctant and rarely simple, a double beat, a self-moved reciprocation. (1945:2)

Dans cet extrait du roman, la ville historique de Montréal, qui se trouve au confluent de deux cultures plus anciennes, participe de cette mythopoétique qui ambitionne de réécrire l'histoire du pays en choisissant des centres. Il convient de rappeler que le Canada au XIX ${ }^{e}$ siècle reste marqué par des cultures régionales et que l'idée de nation ne fera son chemin que progressivement à partir de la naissance de la confédération en 1867. Two Solitudes (1945), une saga familiale qui retrace l'histoire des deux familles Tallard et Methuen, est l'occasion pour lui de construire un mythe «local» qui, selon Bakhtine, figure parmi les diverses manières de faire sens de l'espace et d'expliquer la genèse d'une géographie (Bakhtin, 1981 : 189). La genèse de cet espace centralisé et national dépend de la rencontre et de la coexistence des deux communautés francophone et anglophone, et le discours du narrateur omniscient se réduit parfois à un commentaire sur l'état de la nation et le besoin de voir les deux communautés se réconcilier. Certains critiques ont noté ce ton didactique de Two Solitudes et les romans qui suivront feront parfois les frais de ce ton jugé excessivement didactique et professoral ${ }^{6}$. Le narrateur omniscient de Two Solitudes produit des discours qui s'apparentent à des essais, et cette passion de l'auteur 
pour cette forme a un impact sur l'ensemble de son œuvre et sur le récit de voyage en particulier.

Dans son introduction à L'Essai : Métamorphoses d'un genre, Pierre Glaudes écrit que l'essai est « une forme ouverte et flexible » qui

n'a pas de domaine culturel mieux circonscrit. Tirant tantôt du côté de la philosophie, du discours magistral ou de la méditation spirituelle, il sait aussi se parer des séductions sophistiques de la conversation, recourir aux jeux poétiques ou fictionnels, s'engager hardiment sur les chemins de la vulgarisation journalistique ou de la polémique. De Montaigne à Addison, de Condillac à Barthes, de Charles Lamb à Valéry, c'est un genre protéiforme qui semble n'avoir pas de frontières. (2002: II)

Dans Seven Rivers of Canada, l'essai prend progressivement le dessus sur le récit de voyage à proprement parler, car le voyage lui-même est progressivement relégué au second plan au fil de l'ouvrage. Le second chapitre consacré au Saint-Laurent commence avec la description du fleuve, ses dimensions, ses affluents et son exploitation en termes de transport maritime de marchandises, tant et si bien que le voyage n'est mentionné que deux pages plus loin où le lecteur le découvre sur un cargo qui part vers l'est du pays. MacLennan procède de la même manière lorsqu'il décrit la Rivière Rouge dans la province du Manitoba. Après toute une série de généralités factuelles et historiques sur la rivière, ce n'est qu'à la fin du texte que le lecteur apprend qu'il s'y est rendu en voiture. Qu'il s'agisse du narrateur ou du voyageur, c'est très souvent le discours qui a tendance à dominer l'intrigue ou le voyage. L'essai est la forme qui lui permet de relayer ses convictions politiques et de partager sa version du mythe national.

Ce mythe national qui prend sa source dans les grands fleuves du Canada, et plus particulièrement dans le Saint-Laurent, résulte de la conviction politique de l'auteur selon laquelle le Canada doit rester un pays fédéral et centralisé. La définition qu'il donne de la nation est très claire à ce sujet ; pour lui, ce qui garantit la cohésion nationale est le besoin de se sentir chez soi et de pouvoir défendre son territoire :

If anyone were to ask me now what is the main common denominator which has held this nation together, I would answer very simply: a desire for a home, and a determination to keep it. Ici, nous sommes chez nous-the phrase is constantly used today in French Canada without sentimentality and without embarrassment. (1963:136)

Dans cet extrait, le mythe national censé expliquer la grandeur d'un pays dans les années 1960 permet de relayer un discours nationaliste qui établit la primauté des deux cultures anglophone et francophone au détriment des autres composantes du pays. Mais c'est sans compter les bouleversements démographiques et culturels de cette décennie qui a été le témoin de l'émergence des voix discordantes des minorités ethniques et culturelles. Dans son travail sur les différences identitaires et culturelles au Canada, Récits nord-américains d'émergence, Winfried Siemerling écrit que les années 1960 révèlent deux tendances au Canada pendant cette période: une tendance à rechercher «une homogénéité nationale de la culture » et une autre tendance à valoriser une "pluralité culturelle » qui s'appuie sur cette même homogénéité (2010 : 210). La lecture de l'œuvre de MacLennan l'inscrit pleinement dans cette première tendance qui vise à garantir une certaine homogénéité nationale et une stabilité politique.

C'est sans doute en raison des convictions politiques de l'auteur que les minorités citées dans le texte restent silencieuses. Pendant son voyage sur le MacKenzie, MacLennan rencontre des autochtones, mais ils peinent à se détacher du paysage. Leur présence est souvent rapportée par les personnes qu'il rencontre dans le nord et elle ne donne lieu à 
aucun commentaire de la part de l'auteur. Entre deux remorqueurs, il est accueilli par six hommes dont quatre sont des «Indiens » aux bras musclés, et l'un des guides autochtones cités un peu plus loin dans le récit d'Alexander MacKenzie, et qui portait le titre de English Chief, semble briller par son manque d'efficacité. Ce n'est que lorsqu'il reprend l'avion vers le sud qu'il voit un autochtone de plus près, mais le passage relève surtout de l'anecdote et décrit un passager qui dort :

He was an Indian who had got on the day before at Fort Smith with a ticket for Hay River on the south shore of Great Slave Lake. Though he had never been in a plane in his life, he immediately fell sound asleep and was still asleep when the plane reached the Wells and the other passengers got out. Nobody noticed him and when he woke in the night the plane doors were locked. Since there was nothing he could do, the man fell asleep again and was still asleep when the rest of us got aboard to fly back south. That Indian, at least, understood that the true North is timeless. $(1963: 62)$

Ce passage montre en effet un «Indien» dénué de parole, d'identité et d'histoire. Bien que cet autochtone soit l'une des rares personnes à comprendre l'atemporalité du nord, il est néanmoins relégué à l'arrière-plan. Aucun des passagers ne le remarque ; il disparaît dans le paysage et devient invisible. Un tel regard est en partie imputable à ses convictions humanistes qui, selon Linda Hutcheon, prônent l'ordre, l'unité, l'autorité et l'universalité (1988: 57) au détriment des différences culturelles. Le récit censé ordonner et unifier un espace national ne laisse manifestement que peu de place aux particularités et à l'hétérogénéité.

Il en va de même pour les Highlanders venus d'Écosse. Tout en étant fier de ses origines écossaises et particulièrement élogieux quand il s'agit de décrire le Highlander en tant qu'individu, il écrit néanmoins que les Anglais ont eu raison de les débarrasser de leur système clanique. Dans le récit de son voyage sur la Rivière Rouge, sa position sur le sujet est très claire :

No sane Scotsman could blame the English for treating the Highlanders as they did after the 1745 rebellion. Their blind loyalty to their chiefs, who in turn were blindly loyal to a worthless Pretender, had given the English no choice save to uproot and destroy the ancient clan system. (1963: 116)

Pour MacLennan, une nation moderne qui fédérerait le Canada autour d'un récit commun est incompatible avec l'existence d'anciens systèmes tribaux ou claniques qui menace l'unité. L'idée du progrès qui lui vient principalement d'Europe le contraint à n'envisager la modernité qu'en suivant l'exemple européen. Cependant, cette vision du devenir national n'est pas propre à l'auteur, et Bill Ashcroft, Gareth Griffiths et Helen Tiffin ont écrit à juste titre que le Canada de cette période cherchait la reconnaissance internationale en adoptant une position plus universaliste qui a eu pour effet d'écarter les différences culturelles (Ashcroft et al., 2002: 35). Son écriture du fleuve, symbole du destin du pays, l'entraîne dans un nationalisme qui exclut les particularismes régionaux, au moins sur le plan politique.

Cette vision de la nation sera bien évidemment contestée par d'autres auteurs qui ne voient pas l'identité canadienne d'une manière aussi homogène. Le premier roman de Robert Kroetsch (1965), But We are Exiles, est publié deux ans plus tard et relate l'histoire de Peter Guy, un pilote de remorqueur sur le fleuve MacKenzie. S'il part dans le nord, c'est surtout pour fuir Michael Hornyak, l'ami qui l'a trahi en ayant une relation avec sa compagne Caroline. Lorsque Hornyak tente de retrouver Guy, il meurt noyé, et le bateau de Guy est choisi pour retrouver le corps. Même si But We are Exiles se conforme à un réalisme qui a fait le succès de MacLennan, le MacKenzie que décrit le narrateur est 
résolument différent. Le fleuve est dangereux, et les paysages n'ont pas la clarté de ceux que décrit MacLennan dans Seven Rivers of Canada. Quand il décrit sa vie sur le MacKenzie, le personnage constate à quel point l'ordre apparent du nord n'est qu'une illusion :

No confusion about who is to do what and who did what. From bunk to gallery to the wheelhouse again, six hours on, six hours off, and always out beyond the wheelhouse the thin band of shoreline and trees, separating water and sky. An order maintained as precariously as that maintained by the hands on the wheel. The chaos held in check... (Kroetsch, $1965:$ 19)

L'ordre qui règne sur le bateau n'est en réalité qu'un semblant d'ordre qui peut être bouleversé à tout moment par les caprices et le chaos du Grand Nord. Les paysages résistent à la description et renvoient aux changements et aux doutes qui submergent le personnage. L'intrigue finit avec un protagoniste qui est perdu au milieu d'un blizzard et qui a finalement échoué dans sa tentative de fuite. Chez Kroetsch, le voyage ne permet pas au voyageur de trouver une vérité en suivant le cours du MacKenzie, car le voyageur, à l'instar du fleuve qu'il décrit, n'est jamais stable.

Cette idée d'instabilité sera exploitée également par des écrivains postmodernes comme Leonard Cohen et Margaret Atwood qui proposeront une écriture qui tient davantage compte de l'histoire des communautés autochtones, de la pluralité culturelle apportée par l'immigration et de la particularité de l'expérience individuelle. Beautiful Losers, que Cohen publie en 1966, est une métafiction postmoderne dont le narrateur anonyme, historien et époux d'une femme en partie autochtone, cherche à percer les secrets de Catherine Tekakwitha, une Iroquoise convertie au christianisme au XVII ${ }^{\mathrm{e}}$ siècle. Dans la même veine, le second roman de Margaret Atwood, Surfacing (1972), relate l'histoire d'une narratrice anonyme qui doit retourner dans le Québec de son enfance pour y retrouver ses souvenirs et son identité perdus. Le lecteur découvre à la fin du roman que la narratrice avait dû refouler le traumatisme de son avortement forcé pour survivre. Ce n'est qu'au contact d'une nature labyrinthique et changeante dans le nord du Québec que ses souvenirs referont surface. Rétrospectivement, l'œuvre de MacLennan peut paraître passée de mode par rapport aux nouvelles problématiques identitaires que décrivent ces écrivains. Tout comme ses romans, ses récits de voyage illustrent bien l'ambition didactique et les convictions humanistes de l'auteur. Il ne propose pas de nier le désordre du monde, il cherche, peut-être à tort, à y remédier et à souligner son engagement auprès de la cité. Finalement, et indépendamment des formes et des genres, tous ces auteurs ne cherchaient-ils pas à écrire leur désir de stabilité dans une société en mouvement et bouleversée par les migrations, les nouveautés et les questionnements?

\section{BIBLIOGRAPHIE}

ASHCROFt Bill, GriffithS Gareth \& TIFFIN Helen (2002), The Empire Writes Back: Theory and Practice in Post-Colonial Literatures ( $2^{\mathrm{e}}$ edition), Londres \& New York : Routledge.

ATwOoD Margaret (1972), Surfacing, Toronto : Virago. 
BAKHTIN Mikhail (1981), The Dialogic Imagination (M. Holquist, dir., C. Emerson \& M. Holquist, trad.), Austin : University of Texas Press.

BURKE Edmund (1998), A Philosophical Enquiry into the Origin of our Ideas of the Sublime and Beautiful [1757] (A. Phillips, dir.), Oxford : Oxford University Press.

COCKBURN Robert H. (1967), recension de Return of the Sphinx, Dalhousie Review, 47(3), 435-439.

COHEN Leonard (1966), Beautiful Losers, New York : Vintage.

Deleuze Gilles \& GUATTARI Félix (1980), Capitalisme et schizophrénie 2 : Mille plateaux, Paris : Minuit.

ELIADE Mircea (1963), Aspects du mythe, Paris : Gallimard.

GLAUDEs Pierre [dir.] (2002), L'Essai : Métamorphoses d'un genre, Toulouse : Presses Universitaires du Mirail.

GRACE Sherrill E. (2001), Canada and the Idea of North, Montréal et Kingston : McGill-Queen's University Press.

GuYoT Alain (2012), Analogie et récit de voyage : voir, mesurer, interpréter le monde, Paris : Classiques Garnier.

HUTCHEON Linda (1988), A Poetics of Postmodernism: History, Theory, Fiction, Londres et New York : Routledge.

InNIS Harold A. (1950), Empire and Communications, Oxford : Clarendon Press.

KROETSCH Robert (1977), But We are Exiles [1965], Toronto : Macmillan.

KROETSCH Robert (1995), A Likely Story: The Writing Life, Red Deer : Red Deer College Press.

MACLENNAN Hugh (1935), « Roman History and To-day », Dalhousie Review, 15(1), 67-78.

MACLENNAN Hugh (1963), Seven Rivers of Canada, Toronto : MacMillan.

MACLENNAN Hugh (1998), Two Solitudes [1945], Toronto : Stoddart.

Moodie Susanna (2005), Roughing It in the Bush [1852], New York : Cosimo Classics.

NEW William H. (1995), « The Great River Theory: Reading MacLennan and Mulgan », Essays on Canadian Writing, 56 (automne), 162-182.

SAYRE Robert (2015), « Alexander MacKenzie's Voyages: Indians, the Fur Trade and Northwest Expansion », Représentations, 44-58, <representations.u-grenoble3.fr/IMG/pdf/5-

sayre_mckenzie_final_def.pdf>.

SIEMERLING Winfried (2010), Récits nord-américains d'émergence : Culture, écriture et politique de re/ connaissance, Patricia Godbout (trad.), Laval : Presses universitaires de Laval.

Soja Edward W. (1989), Postmodern Geographies: The Reassertion of Space in Critical Social Theory, Londres et New York: Verso.

TVERDOTA György [dir.] (1994), Écrire le voyage, Paris : Presses de la Sorbonne Nouvelle.

VIVIÈs Jean (1999), Le récit de voyage en Angleterre au XVIII ${ }^{e}$ siècle : de l'inventaire à l'invention, Toulouse : Presses universitaires du Mirail. 


\section{ANNEXES}

André Dodeman est actuellement maître de conférences à l'Université Grenoble-Alpes, à l'UFR de Langues Étrangères et membre de l'unité de recherche ILCEA4. Il a soutenu sa thèse de doctorat en novembre 2008 sous la direction de Mme le Professeur Marta Dvorak (Université Paris 3, Sorbonne Nouvelle), et son travail a porté sur l'œuvre littéraire de l'auteur canadien Hugh MacLennan. Il a publié des articles sur plusieurs auteurs canadiens anglophones et a codirigé deux ouvrages dans le domaine des études postcoloniales : avec Catherine Delmas, Re/membering Place (2013), Bern : Peter Lang ; avec Cyril Besson, Ville et environnement : Regards croisés sur le monde postcolonial (2014), Paris : Michel Houdiard éd.

\section{NOTES}

1. Le terme de fleuve employé ici renvoie aussi bien aux fleuves qu'aux rivières, car, en anglais, le terme "river " permet aussi de désigner les affluents. Alors que le MacKenzie est un fleuve qui termine sa course dans l'Arctique, la rivière des Outaouais (The Ottawa River) reste l'un des principaux affluents du fleuve Saint-Laurent.

2. Hugh MacLennan termine sa thèse de doctorat en 1935 à Princeton. L'auteur y décrit l'évolution sociale et économique de la petite ville égyptienne d'Oxyrhynche au crépuscule de l'empire romain. En 1935, MacLennan accepte, à contrecœur, un poste de professeur de latin et d'histoire au Lower Canada College, près de Montréal.

3. Dans Empire and Communications (1950), Harold Innis traite lui aussi des relations entre les fleuves et la civilisation. Dans son chapitre sur l'Égypte ancienne, il écrit que le Nil a fonctionné comme un principe d'ordre et de centralisation: "The Nile acted as a principle of order and centralization, necessitated collective work, created solidarity, imposed organizations on the people and cemented them in a society » (Innis, $1950: 13$ ).

4. Il s'agit en réalité de la définition que Soja donne de la territorialité en géographie. Dans le texte original, «[t]erritoriality is the more general term and contains hints of such particularized notions as sovereignty, property, discipline, surveillance and jurisdiction. » (Soja, 1989 : 150)

5. Dans un article qu'il publie dans la Dalhousie Review dans les années 1930, intitulé «Roman History and To-day », MacLennan sous-entend que l'histoire est intelligible, car elle est avant tout causale: "Any completed pattern has a meaning; and if that meaning can be isolated and rendered coherent, it must necessarily help to explain our own society to ourselves» (1935: 67). MacLennan reste aussi très marqué par ses lectures, et plus particulièrement par les ouvrages théoriques de G. W. F. Hegel et d'O. Spengler portant sur l'Histoire.

6. C'est le cas de son avant-dernier roman, Return of the Sphinx (1967), qui revient sur le conflit qui oppose les anglophones aux francophones au Canada. Dans la Dalhousie Review, Robert H. Cockburn rédige une recension qui déplore l'excès de ce ton didactique : "The Return of the Sphinx shows that Hugh MacLennan continues to confuse fiction and non-fiction; he still evinces a serious poverty of imagination, still insists upon teaching instead of creating. ", The Dalhousie Review, 47(3), 437-439. 


\section{RÉSUMÉS}

Alors connu au Canada pour les cinq romans qu'il écrit entre 1945 et 1963, Hugh MacLennan ne se cantonne pas pour autant à cette forme. Il choisit en effet l'essai pour relayer ses convictions politiques et artistiques et le récit de voyage pour rassembler un public canadien autour de l'idée d'une nation que seuls les fleuves du pays peuvent traduire sous forme d'image. Cet article montrera tout d'abord de quelle manière l'auteur s'extrait du rôle de l'écrivain engagé qui s'efforce d'éclairer ses lecteurs pour devenir un voyageur qui partage son expérience de terrain et esthétise un paysage alors méconnu du grand public. Le paysage nordique que certains écrivains perçoivent comme chaotique et irrégulier devient, sous la plume de MacLennan, un territoire national ordonné qui invite le lecteur à mieux se l'approprier. Ce travail propose de montrer que le récit de voyage de l'auteur reflète en réalité une nette opposition entre deux conceptions très différentes de la nation: l'une qui s'inspire d'une remise en cause de l'idée traditionnelle de nation et tient compte des changements littéraires et démographiques du pays, et l'autre, celle de MacLennan, qui reste attachée au canon littéraire européen et cherche à subsumer les différences culturelles dans une nation unie.

In the early 1960s, Hugh MacLennan was better known for the novels he wrote between 1945 and 1963, but he seldom refrained from exploring other forms. Indeed, he chose to write essays to express his political and artistic convictions and travel narratives to encourage Canadian readers to embrace the idea of a nation symbolized by the country's great river system. This article will show how the writer changes from the writer engagé who is intent on enlightening his readership to the traveler who shares his experience and aestheticizes landscapes that most Canadians were unfamiliar with at the time. The northern landscapes that some writers considered to be chaotic and irregular would become, in MacLennan's text, a well-ordered national territory that readers would be able to appropriate. This paper proposes to demonstrate that the writer's travel narrative illustrates a sharp opposition between two very different definitions of the nation: one that challenges more traditional representations of the nation and considers the various literary and demographic shifts and trends of the period, and another, largely supported by MacLennan, that is inspired by the European literary canon and subsumes cultural differences under the idea of a united nation.

\section{INDEX}

Mots-clés : Canada, paysage, littérature, voyage, nation, fleuve

Keywords : Canada, landscape, literature, travel, nation, river

\section{AUTEUR}

\section{ANDRÉ DODEMAN}

Maître de conférences à l'Université Grenoble-Alpes (ILCEA4) 